\title{
Dendritoma vaccination combined with low dose interleukin-2 in metastatic melanoma patients induced immunological and clinical responses
}

\author{
YANZHANG WEI ${ }^{1,3}$, ROBERT P. STICCA ${ }^{1,4}$, LILLIA M. HOLMES ${ }^{1}$, KELLY E. BURGIN ${ }^{1}$, \\ JINHUA LI ${ }^{1}$, JANE WILLIAMSON ${ }^{1}$, LYNDON EVANS ${ }^{2}$, SAMUEL J. SMITH ${ }^{1}$, \\ JOSEPH J. STEPHENSON ${ }^{2}$ and THOMAS E. WAGNER ${ }^{1,3}$
}

\author{
${ }^{1}$ Oncology Research Institute and the Cancer Treatment Center, Greenville Hospital System; ${ }^{2}$ Cancer Centers \\ of the Carolinas, Greenville; ${ }^{3}$ Department of Biological Sciences, Clemson University, Clemson, SC, USA
}

Received August 29, 2005; Accepted October 17, 2005

\begin{abstract}
A pilot clinical trial using dendritomas, purified hybrids from the fusion of dendritic/tumor cells combined with a low dose of IL-2, in metastatic melanoma patients was conducted in order to determine its safety and potential immunological and clinical responses. Ten metastatic melanoma patients were enrolled into this study. Dendritoma vaccines were created by fusing dendritic cells stained with green fluorescent dye with irradiated autologous tumor cells stained with red fluorescent dye and purifying the hybrids using immediate fluorescent-activated cell sorting. Initial vaccine was given subcutaneously and followed by IL-2 in serially elevated doses from 3-9 million units $/ \mathrm{m}^{2}$ for 5 days. Repeated vaccinations were administered without IL-2, at 3 -month intervals for a maximum of 5 times. Immune reactions were measured by the increase of interferon- $\gamma$ (IFN- $\gamma$ ) expressing $\mathrm{T}$ cells. Vaccine doses ranged from 250,000 to $1,000,000$ dendritomas. There was no grade 2 or higher toxicity directly attributable to the vaccine. All patients experienced toxicity due to IL-2 administration (9-grade 2, 3-grade 3, 1 -grade 4$)$. Eight of nine evaluable patients demonstrated immunologic reactions by increased IFN- $\gamma$ expressing $\mathrm{T}$ cells. One patient developed partial response at 12 weeks after the first vaccine. Nine months later, this patient achieved a complete response. In addition, two patients had stable disease
\end{abstract}

Correspondence to: Dr Thomas E. Wagner, Oncology Research Institute, Greenville Hospital System, 900 West Faris Road, Greenville, SC 29605, USA

E-mail: twagner@ghs.org

Present address: ${ }^{4}$ Department of Surgery, University of North Dakota School of Medicine and Health Sciences, 501 North Columbia Road, Grand Forks, ND 58203, USA

Key words: dendritic cell, dendritoma, tumor, fusion, immunotherapy, melanoma, clinical study for 9 and 4 months, respectively; one patient had a mixed response. Our findings demonstrated that dendritoma vaccines with a low dose of IL-2 can be safely administered to patients with metastatic melanoma and induce immunological and clinical responses.

\section{Introduction}

Metastatic malignant melanoma carries a dismal prognosis, and few therapies have been found to produce responses with improvement in overall survival. Response rates to systemic chemotherapeutic agents are marginal, and median survival from time of diagnosis is about 6-12 months. In selected cases with single or limited distant metastases surgical resection has yielded cure rates of $15-25 \%$, but most patients develop disseminated metastases and succumb to the disease. Despite many years of intensive research, there are few therapeutic options for the management of metastatic malignant melanoma.

Dendritic cells (DCs) are professional antigen presenting cells (APCs) that play a vital role in stimulating immune responses. DCs not only activate naïve CD4+ T helper cells, but also stimulate unprimed CD8+ T lymphocytes (1-4). Because of these characteristics, DCs have been widely studied as antigen presenting cells for cancer immunotherapy $(5,6)$. On the other hand, it has been demonstrated by our laboratory and others that tumor cells persist partly because they have selected mutations that partially or completely protect them from being destroyed by the immunosurveillance (7-11). The in vitro loading of DCs with tumor antigens is becoming feasible and useful. Initially, DCs were loaded with tumor antigens or whole tumor lysate by pulsing and based on these procedures, several clinical trials have been conducted (12-18). However, it is known that the antigen presenting system of an APC works more effectively and efficiently when the protein/antigen is synthesized inside the cell rather than outside the cell, especially when cellular immune responses are considered (19). Therefore, a number of laboratories have attempted to introduce specific tumor antigens into dendritic cells using gene transfer methods (20-23). However, this is also fraught with many disadvantages, including: 1) the limited ability to identify all of the important specific tumor antigens; 2) the 


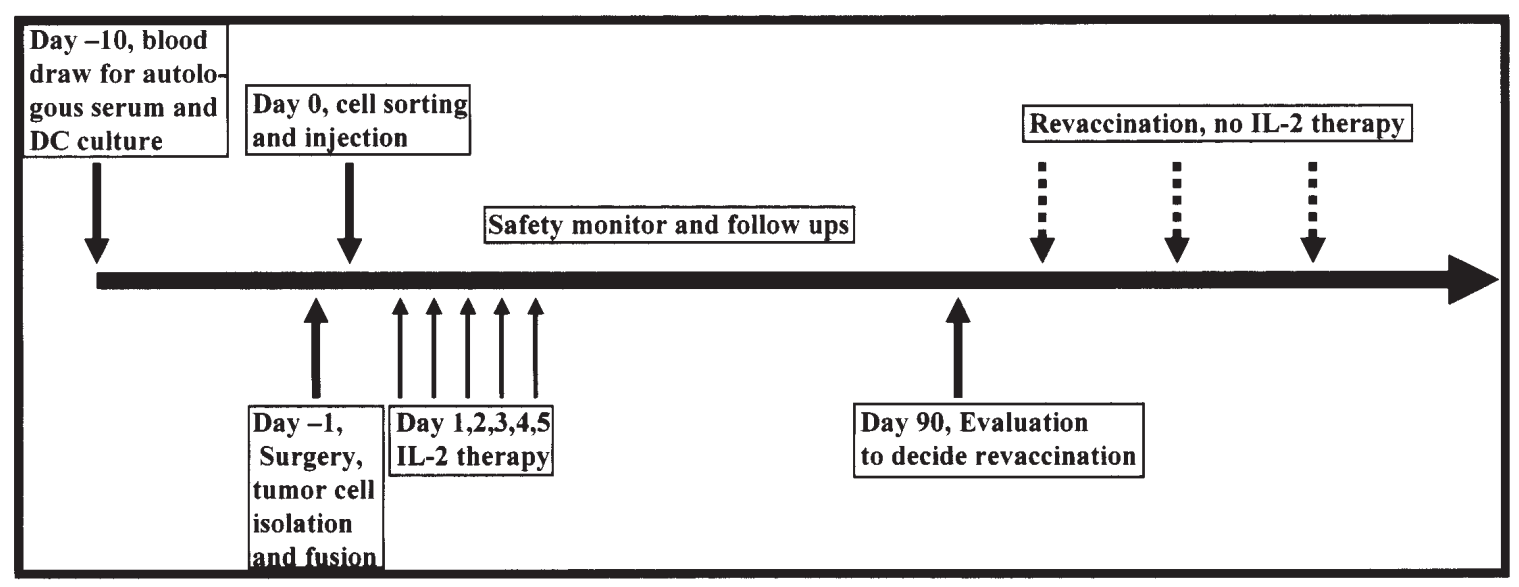

Figure 1. Diagram of the trial design.

limited ability to map the genes of the specific tumor antigens; 3 ) only one or a small number of the known tumor antigen genes can be introduced into the dendritic cell; and 4) the process is time-consuming and cumbersome. Given these disadvantages, the ideal solution would be to introduce the entire tumor genome into the dendritic cell so that the entire portfolio of tumor genes would be expressed within the dendritic cell. The ideal or obvious way to do this would be to create a hybrid of the dendritic and tumor cell. Several studies have shown encouraging results by fusing DCs and tumor cells both in animal studies and in early clinical trials (24-34). However, the purification of the fused hybrid cells from fusion mixtures has proven difficult because of the lack of feasible selection markers. Therefore, in these fusion studies either the entire fusion mixture or loosely purified cells were used $(24,25)$. Furthermore, traditional means of selecting fused from unfused cells, such as in hybridoma generation, involves culture and selection in culture. Unfortunately, such a selection in culture may only result in retaining a portion of the antigenic diversity of a tumor yielding a vaccine that may not be effective against some aspects of the heterogeneous cellular population of a tumor.

We hypothesized that the use of highly purified hybrid cells from DC-tumor cell fusion would be more effective than the entire fusion mixture in stimulating tumor cell specific antitumor immunity. Additionally, it was our goal to generate this highly purified cell population instantly and without culture. We developed a novel technique by which hybrid cells can be easily purified from a fusion mixture (35). By using this technique, hybrid cells were instantly purified from fusions between DCs and tumor cells and named dendritomas. Dendritomas retained the characteristics of the tumor cell as well as the ability of the DC to act as an effective APC. Our animal studies have confirmed that the purified dendritomas are better activators than fusion mixtures in stimulating tumor-specific anti-tumor immunity (36). In vitro studies using human cells also showed that dendritomas made from patients' peripheral blood DCs and autologous primary tumor cells effectively activated autologous $\mathrm{T}$ cells to lyse autologous tumor cells. Therefore, we reasoned that the introduction of these dendritomas into patients may allow them to effectively present the tumor antigens to the patients' immune cells and to stimulate the generation of anti-tumor immune responses. Also, because these dendritomas represent the entire diversity of tumor cell types, we anticipated that they might stimulate a more complete immune response.

Based on the results from animal studies and our in vitro human study, we designed a pilot clinical trial to determine the feasibility of a treatment regimen using dendritomas as a vaccine for immunotherapy in patients with metastatic melanoma. In order to boost the immune responses generated by dendritoma vaccine, if there were any, low doses of IL-2 were followed after the first vaccine. The primary objective of this study was to evaluate safety of the vaccine by assessing adverse events; the secondary objective was to assess immune responses by measuring the increase of IFN- $\gamma$ expressing $\mathrm{T}$ cells and clinical responses.

\section{Patients and methods}

Patient selection and study design. All patients had histologically confirmed metastatic malignant melanoma and an expected survival of 3-6 months. An ECOG performance status of $\leq 3$ was necessary to enter the trial. Inclusion criteria included adequate pulmonary function (FEV1 $>25 \%$ predicted or DLCO $>25 \%$ predicted), adequate cardiac function, serum creatinine $<1.6, \mathrm{Hgb}>9.0, \mathrm{WBC}>3,000$, platelet count $>100,000$ and no prior history of a seizure disorder. Previous treatment with chemotherapy or immunosuppressive agents had to be discontinued at least 30 days prior to vaccination. Previous treatment with other forms of immunotherapy had to be discontinued 6 months prior to vaccination unless there was documented progression of the disease. CNS metastases were allowed, and there were no limitations on tumor location or volume. It was necessary for each patient to have a tumor with a volume $>1 \mathrm{~cm}^{3}$ available for surgical excision (for vaccine preparation) and measurable residual disease for evaluation of response. Bidirectional measurements of residual disease were recorded from a maximum of 4 index lesions, either by direct measurement for cutaneous lesions or computed tomography (CT) measurements for visceral lesions. Tumor volumes were calculated from these 
Table I. Clinical characteristics of patients.

\begin{tabular}{|c|c|c|c|c|c|}
\hline Patient No. & Age & Sex & Previous therapies ${ }^{\mathrm{a}}$ & Disease stage at entry of this trial & Sites of tumors at entry of this trial \\
\hline 1 & 66 & $\mathrm{~F}$ & $\mathrm{~S}, \mathrm{Ch}$ & IV & $\mathrm{Br}, \mathrm{L}, \mathrm{Li}, \mathrm{Sc}, \mathrm{Ly}$ \\
\hline 2 & 61 & $\mathrm{~F}$ & $\mathrm{R}, \mathrm{Ch}, \mathrm{S}$ & IV & $\mathrm{L}, \mathrm{Li}, \mathrm{B}$ \\
\hline 3 & 45 & $\mathrm{M}$ & $\mathrm{S}$ & IV & L, Li, Ia, Sc, Lr \\
\hline 4 & 68 & $\mathrm{M}$ & S. Ch & IV & $\mathrm{C}, \mathrm{Sc}, \mathrm{Ly}, \mathrm{Li}$ \\
\hline 5 & 84 & M & $\mathrm{S}$ & IV & $\mathrm{C}, \mathrm{Lr}, \mathrm{Ly}$ \\
\hline 6 & 51 & $\mathrm{~F}$ & $\mathrm{~S}, \mathrm{Ch}$ & IV & Co, Ly \\
\hline 7 & 74 & M & $\mathrm{S}$ & IV & L, Br, Li C, Sc, Ly, Lr \\
\hline 8 & 32 & M & $\mathrm{S}, \mathrm{Ch}$ & IV & Sc, Ly \\
\hline 9 & 63 & $\mathrm{~F}$ & $\mathrm{~S}, \mathrm{Ch}$ & IV & $\mathrm{Sc}, \mathrm{L}$ \\
\hline 10 & 73 & M & $\mathrm{S}, \mathrm{R}$ & IV & $\mathrm{Sc}, \mathrm{C}, \mathrm{Ly}$ \\
\hline
\end{tabular}

${ }^{a}$ S, surgery; Ch, chemotherapy; R, radiation. ${ }^{\text {bB }}$, bone; L, lung; Li, liver; Lr, local recurrence; Br, brain; Ly, lymph nodes; Sc, subcutaneous; Ia, intraabdominal; $\mathrm{C}$, cutaneous; Co, colon.

measurements for comparison with post treatment evaluations. Informed consent was obtained from each patient prior to entry into the trial. Human investigations were approved by the Institutional Review Committee of the Greenville Hospital System, in accord with an assurance filed with and approved by the Department of Health and Human Services. Production and use of vaccines in this protocol was approved and monitored by the Food and Drug Administration (IND 8851). The trial design is diagramed in Fig. 1.

Tumor and dendritic cell preparation. Autologous serum was used for preparation and maintenance of each patient's tumor cells and DCs. Blood $(200 \mathrm{ml})$ was withdrawn from the patient at least 2 weeks prior to initial vaccination. Serum was prepared from it using standard techniques. Prior to use, the serum was heat-inactivated by incubating at $56^{\circ} \mathrm{C}$ for $30 \mathrm{~min}$. This autologous serum comprised $10 \%$ by volume of the media used for maintenance of patient's tumor cells and DCs.

Mature DCs were generated from the patient's peripheral blood monocytes (PBMCs). Sodium-heparinized peripheral blood $(300 \mathrm{ml})$ was obtained from the patient. PBMC's were isolated by Ficoll-Paque Plus gradient (Amersham Biosciences). Monocytes were then isolated from the PBMC's by panning for 3-4 $\mathrm{h}$ in petri dishes. The purified monocytes were then cultured in complete DC medium (RPMI-1640 $+10 \%$ human serum $+800 \mathrm{U} / \mathrm{ml} \mathrm{GM-CSF}+1000 \mathrm{U} / \mathrm{ml}$ IL-4) at $37^{\circ} \mathrm{C}, 5 \% \mathrm{CO}_{2}$ for $7-10$ days to generate dendritic cells. The DC medium was refreshed every 3-4 days. Dendritic cells were matured by adding $100 \mathrm{ng} / \mathrm{ml}$ of $\mathrm{TNF} \alpha$ and incubating for 24-48 h. Tumor tissue was obtained by direct biopsy or excision of metastatic lesions in each patient. The tumor specimens were removed from the patients under sterile conditions and processed as follows: After separating fat and extraneous tissue away from the tumor tissue, the tumor was cut into small chunks and put into a T75 flask containing $20 \mathrm{ml}$ of RPMI-1640, $1.5 \mathrm{mg} / \mathrm{ml}$ collagenase type VIII (Sigma), $26 \mu \mathrm{g} / \mathrm{ml}$ pulmozyme (Genentech). This solution was rocked for $1-2 \mathrm{~h}$ at $37^{\circ} \mathrm{C}$ and the cell suspension was then filtered through a $40 \mu \mathrm{m}$ cell strainer (Falcon Cat No. 2340). After 2 washes with $1 \mathrm{X}$ PBS, the cells were resuspended in ACK lysing solution $\left(0.15 \mathrm{M} \mathrm{NH}_{4} \mathrm{C} 1,1 \mathrm{mM} \mathrm{KHCO}_{3}, 0.1 \mathrm{mM}\right.$ $\mathrm{Na}_{2}$ EDTA, $\mathrm{pH}$ 7.3) and incubated for $5 \mathrm{~min}$ at room temperature to lyse the red blood cells. After centrifugation, the cells were re-suspended in complete tumor cell media (DMEM, $10 \%$ autologous serum, $200 \mu \mathrm{g} / \mathrm{ml}$ gentamycin) until fusion.

Staining, fusion and sorting. DCs were stained green using the PKH2-GL fluorescent dye (Sigma) and tumor cells were stained red using the PKH26 fluorescent dye (Sigma) according to the manufacturer's instructions. Then the tumor cells were exposed to a single dose of $50 \mathrm{~Gy}$ irradiation, sufficient to render the cells replication incompetent. The green DCs and the red tumor cells were fused at a 1:1 ratio according to a standard PEG fusion protocol. The fusion mixtures were incubated in complete DC medium overnight in a humidified $37^{\circ} \mathrm{C}, 5 \% \mathrm{CO}_{2}$ incubator. The next day the cells were analyzed and hybrid cells (dendritomas) were sorted according to the presence of dual colors (red and green) using the FACS Vantage SE (BD Biosciences).

Treatment. Vaccines consisted of 250,000-1,000,000 (mean $813,000)$ dendritomas. The number of dendritomas in each patient's vaccine varied depending on the yield from the culture and fusion process. After irradiation at $100 \mathrm{~Gy}$, these dendritomas were then re-suspended in 2-3 $\mathrm{ml}$ of normal saline (NS) and within $24 \mathrm{~h}$ of re-suspension were injected subcutaneously into the patient immediately adjacent to a lymph node basin. One day after dendritoma injection, IL-2 was administered by subcutaneous injection at a starting dose of $3 \mathrm{mIU} / \mathrm{m}^{2} /$ day with sequentially increasing doses by $3 \mathrm{mIU} /$ $\mathrm{m}^{2} /$ day to a maximum dose of $9 \mathrm{mIU} / \mathrm{m}^{2} /$ day for a total of 5 days (Fig. 1). Each patient was premedicated $1 \mathrm{~h}$ prior to IL-2 injection with claritin (10 mg), and celebrex (100 mg) or relafen $(1000 \mathrm{mg})$. Patients were revaccinated every 3 months depending on the availability of dendritomas and tumor cells. Revaccination was administered in similar fashion as the initial vaccination with the exception that IL-2 was not given. Patients received a maximum of 5 vaccinations depending on their disease progression and availability of vaccine. 

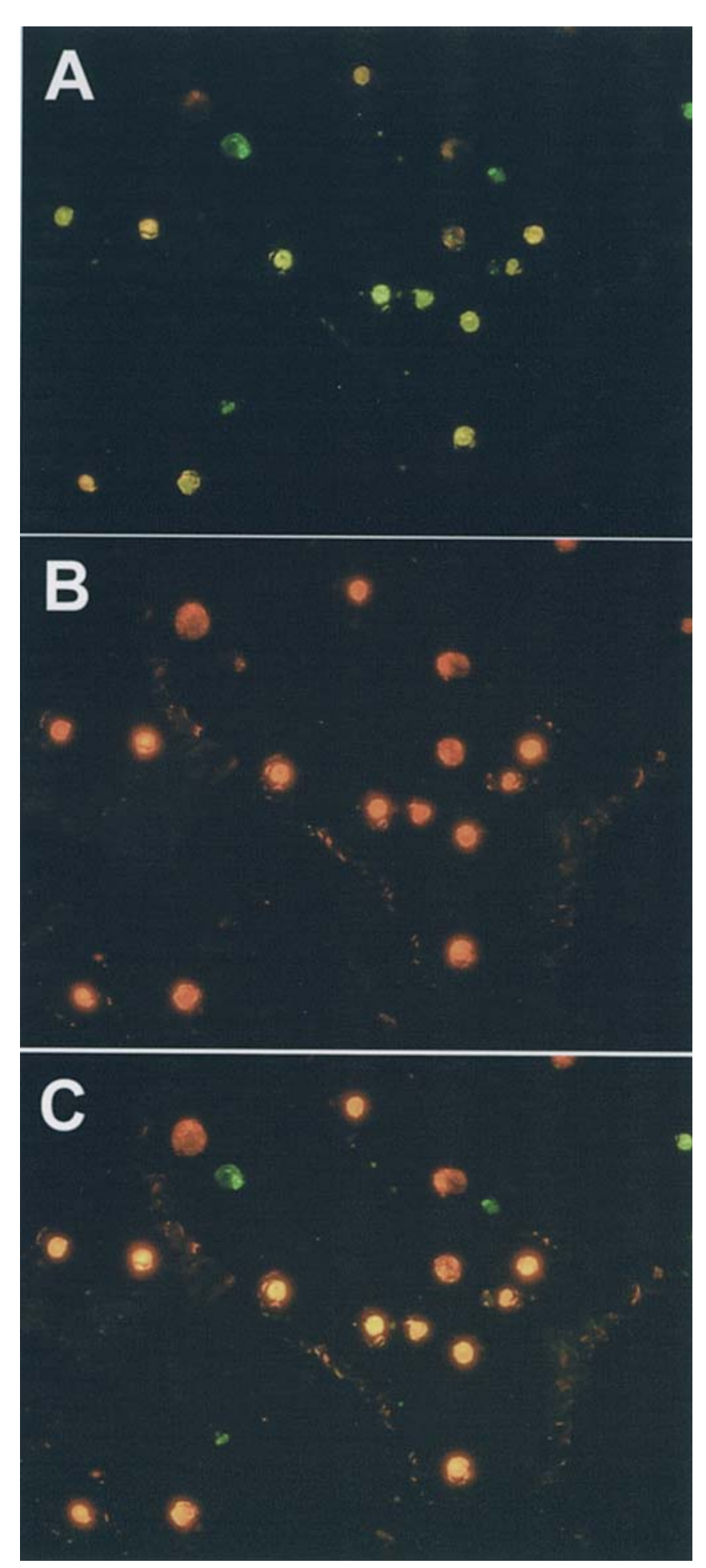

Figure 2. Dendritomas. Freshly sorted dendritomas were observed under fluorescent microscope. The same field was pictured with a green filter (A), red filter (B), and green and red filter (C).

Follow-up. All patients were followed closely at prescribed intervals throughout recurrence or progression, if present, until death. They were evaluated closely for pulmonary, renal, hepatic, gastrointestinal, cardiac, autoimmune or other toxicities in addition to evidence of regression or progression of their index lesions. Immediately post vaccination patients were followed at days 1 and 3 with complete blood count (CBC), creatinine, liver function tests (LFTs), and clinical examinations. The 2-week and 1-month follow-ups included clinical examination, $\mathrm{CBC}$, electrolytes, BUN, and creatinine. CT scans of the head, chest, abdomen, and pelvis were obtained at 3-month intervals prior to revaccination.

$I F N-\gamma$ assay. Before the first vaccine, 2 and 4 weeks after the first vaccine, and 1 and 4 weeks after vaccination, blood samples were taken from the patients and used to perform the intracellular interferon- $\gamma$ analysis using anti-human IFN- $\gamma$ FastImmune $^{\mathrm{TM}}$ CD4 Intracellular cytokine detection kit (BD Biosciences). Briefly, $1.5 \mathrm{ml}$ whole blood from each sample were distributed into 3 conical tubes (each $0.5 \mathrm{ml}$ ) labeled as unactivated (negative control), activated (experiment), and SEB (Staphylococcal Enterotoxin B, positive control), respectively. CD28/CD49 (5 $\mu 1$ ) from BD FastImmune intracellular cytokine detection kit was then added into each tube. Autologous tumor lysates $(5 \mu 1)$ and SEB $(2 \mu 1)$ were added into the 'activated' tube and the 'SEB' tube, respectively. The tubes were vortexed and incubated at $37^{\circ} \mathrm{C}$ for $2 \mathrm{~h}$. BFA solution $(10 \mu \mathrm{l})$ from the kit was added into each tube and the tubes were further incubated for $4 \mathrm{~h}$. After the 4-h incubation, $50 \mu 1$ EDTA solution was added into each tube. The red blood cells in the tubes were lysed by adding $5 \mathrm{ml}$ lysing solution (from the kit) into each tube and incubating at room temperature for $10 \mathrm{~min}$. After 2 washings with staining buffer $\left(0.1 \%\right.$ BSA and $\left.0.1 \% \mathrm{NaN}_{3} \mathrm{PBS}\right)$, the cells were stained with anti-CD4-PerCP-Cy5.5 or anti-CD8-PerCP-Cy5.5 and CD69-PE antibodies. After surface marker staining, the cells were permeablized and stained with anti-IFN- $\gamma$-FITC antibody. The cells were then analyzed on a FACS Calibur with CellQuest software (BD Biosciences). Because of the addition of marker CD69, an activated T cell marker, we were able to analyze IFN- $\gamma$ production by activated CD4+ T cells and CD8+ $\mathrm{T}$ cells.

\section{Results}

Patient characteristics. Ten patients with stage IV melanoma were enrolled in this study. Their clinical characteristics are shown in Table I. All patients had histologically confirmed metastatic melanoma, which was metachronous in all cases. The average time interval between initial diagnosis of malignant melanoma and development of metastasis was 45.5 months. Initial stage at diagnosis was stage $\mathrm{I}$ in one patient, stage II in 5 patients, stage III in 3 patients, and stage IV in one patient. Seven patients had undergone previous therapy for metastatic melanoma that included chemotherapy in 6 patients and immunotherapy in one patient.

Vaccine production and safety. Autologous DCs stained green were fused with irradiated, fresh (or frozen) tumor cells stained red using PEG. The fusion efficiency was from 5 to $15 \%$. After overnight incubation, the hybrid cells were purified using FACS vantage sorting according to the dual colors. Fluorescent microscopic analysis of the sorted cells demonstrated that they are hybrid cells possessing both green and red colors (Fig. 2). For this pilot study, 23 dendritoma vaccines ranging from 250,000 to $1,000,000$ dendritomas were produced and successfully administered for the ten patients (Table II). The safety data, based on observation of the 10 study patients, 
Table II. Treatment protocol and adverse events.

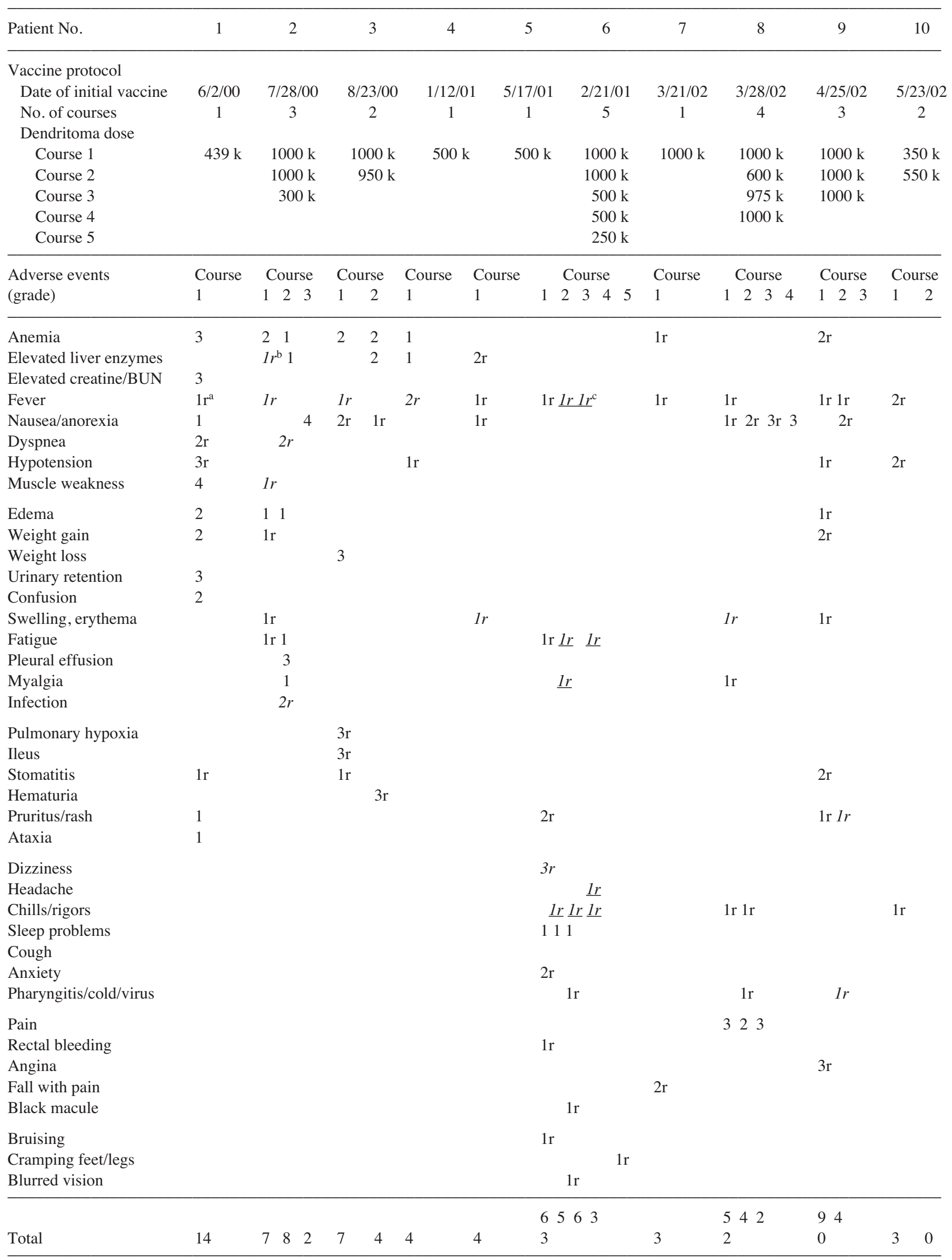

${ }^{a}$ r, adverse event resolved; ${ }^{b}$ possible vaccine-related adverse events are italicized; ${ }^{c}$ vaccine-related adverse events are underlined. 
Table III. Clinical summary of patients.

\begin{tabular}{rcccc}
\hline Patient No. & Date of first vaccine & No. of vaccines & $\begin{array}{c}\text { Disease staging 12 weeks post } \\
\text { 1st vaccine and last/month }\end{array}$ & Days from 1st vaccine to death \\
\hline 1 & & 1 & DFD & 19 \\
2 & Jun-00 & 3 & SD/9 & 363 \\
3 & Jul-00 & 2 & PD & 267 \\
4 & Aug-00 & 1 & PD & 85 \\
5 & Jan-01 & 1 & PD & 94 \\
6 & May-01 & 5 & PR/9 & DFD \\
7 & Feb-02 & 1 & PD/MR & 45 \\
8 & Mar-02 & 4 & SD/3 & 340 \\
9 & Mar-02 & 3 & PD & 254 \\
10 & Apr-02 & 2 &
\end{tabular}

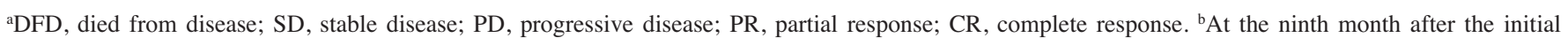
vaccine, this patient had a mixed response.

revealed that the vaccine can be administered without serious adverse events occurring that are attributable to the vaccine. The most common adverse events were expected events associated with IL-2, including grade 1 and 2 fever, grade 1 and 2 chills and rigors, grade 2 and 3 anemia, grade 2 rash, and grade 2 stomatitis. One patient experienced grade 3 angina on the first day of IL-2 administration. The pain resolved, and all cardiac tests were normal. Only one patient experienced grade 1 toxicity (transient chills, fever and arthralgias) attributable to the vaccine (Table II). This same patient experienced a grade 3 toxicity (dizziness) after her fifth vaccine injection, which could possibly be attributed to the vaccine. Other possible toxicities attributable to the vaccines include grade 1 swelling/erythema at cutaneous sites, grade 1 rash and grade 1 pain. All possible toxicities related to the vaccine resolved.

Dendritoma vaccine increases tumor cell specific IFN- $\gamma$ expressing T cells. IFN- $\gamma$ expressing CD4+ or CD8+ T lymphocytes were measured in peripheral blood mononuclear cells (PBMCs) isolated from the patient pre-vaccination, 1, 2 or 4 weeks post first vaccination and 1 and 4 weeks post second, third, or fourth vaccinations using the FastImmune CD4 or CD8 intracellular cytokine detection kits with anti-human interferon- $\gamma$. The PBMCs were unstimulated as negative controls, stimulated with patient's autologous tumor lysate, or stimulated with staphylococcal enterotoxin B as positive controls. Staining of the PBMCs was performed according to the manufacturer's directions. Analysis of CD4+ or CD8+ T lymphocytes expressing IFN- $\gamma$ was performed on a FACS calibur (BD Biosciences). Eight of the nine evaluable patients showed increased IFN- $\gamma$ expressing CD4+ T cells at least at one time point post vaccine compared to prior to vaccine (Fig. 3A). Six of seven analyzed patients showed increased IFN- $\gamma$ expressing CD8+ T cells at least at one time point post vaccine compared to prior to vaccine (Fig. $3 \mathrm{~B}$ ). The increases took place at different time points in different patients. Some patients' IFN- $\gamma$ expressing $\mathrm{T}$ cells increased 2 weeks after the first vaccination (Patient No. 2, 5, and 6 for CD4+ T cells; Patient No. 2 and 7 for CD8+ T cells), while some other patients' IFN- $\gamma$ expressing $\mathrm{T}$ cells increased later (Patient No. 3, 4, 8, 9 and 10 for CD4+ T cells; Patient No. 6, 8,9 , and 10 for CD8+ T cells). In one patient (No. 8) the increase of INF- $\gamma$ expressing CD4+ T cell occurred prior to the fourth vaccination. When compared to the unstimulated cells (open bars), most of the increases of IFN- $\gamma$ expressing T cells are tumor lysate specific (solid bars).

Clinical responses. One patient died 19 days after the first vaccination due to rapid disease progression. Thus, 9 patients were able to be evaluated for clinical response. One patient (No. 6) who received a total of 5 vaccinations had a partial response $(50 \%$ decreases in tumor size) 3 months after the first vaccine. At 6 months after the first vaccine, the patient's tumor regressed by $68 \%$. At 14 months, she developed a complete response and is still tumor-free after receiving 5 vaccinations at 3 month intervals and the 18 months of followup without having any additional therapy. This patient had a grade 3 vertigo (dizziness) possibly due to the vaccine. At 3 months after vaccination, Patient No. 2's disease remained stable for 9 months and the disease of Patient No. 9 remained stable for 4 months. Patient No. 8 developed a mixed response 9 months after vaccination with his subcutaneous nodules completely regressed and regression of the inguinal mass, while other areas were progressing. The clinical data are summarized in Table III.

\section{Discussion}

Despite years of research and attempts at effective treatment, there remain few therapeutic options for metastatic malignant melanoma, except surgical resection. Immunotherapy has held promise for many years, but an effective route of therapy has remained elusive despite many different trials with vaccines and other biologic agents (6).

DC and tumor cell fusion as a vaccine has been examined in other trials and the results are encouraging (24-34). Since there was no convenient method to purify the fused hybrids, 
A
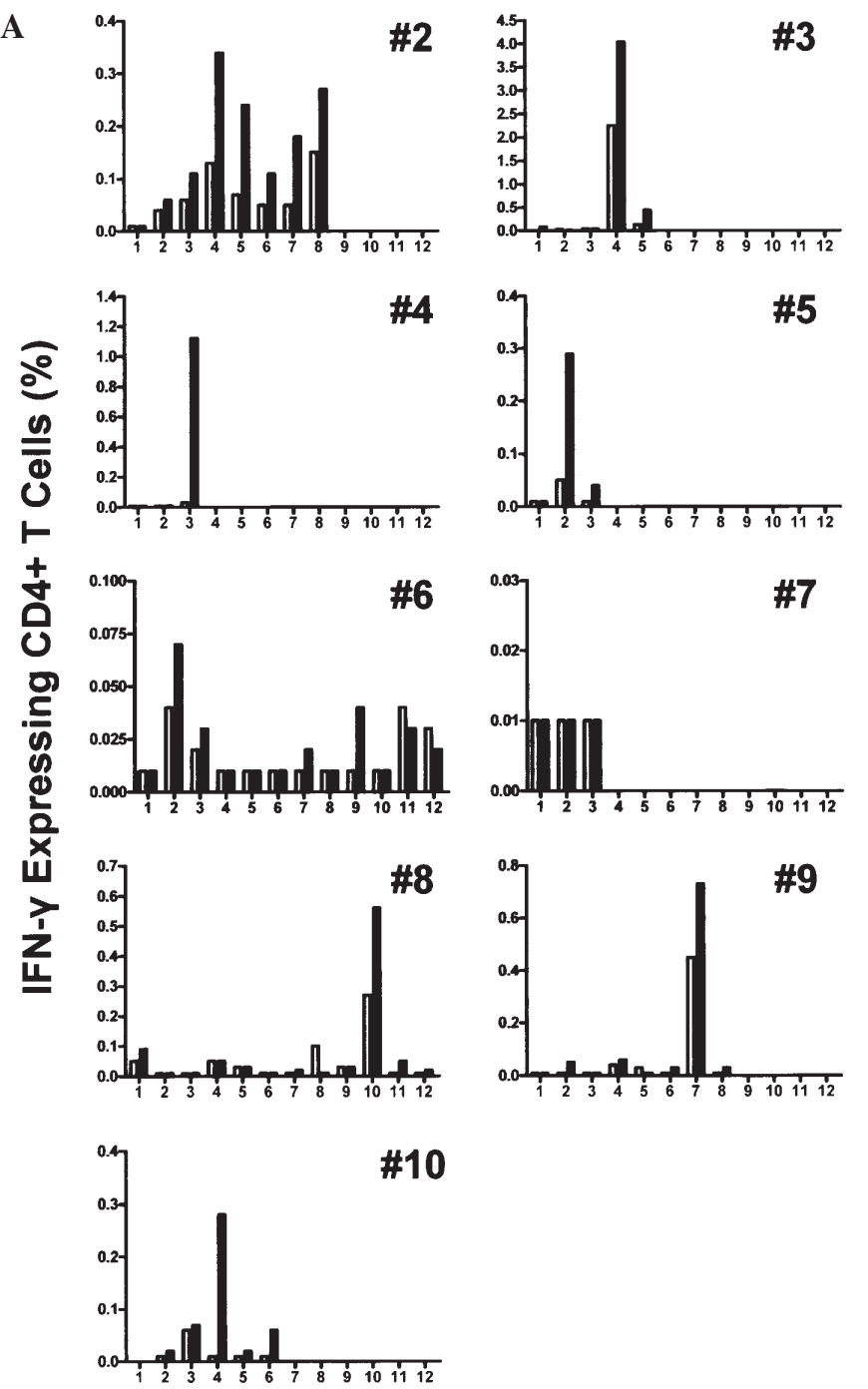

B
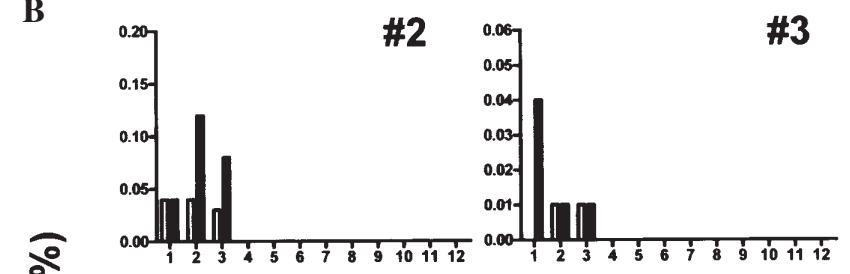

\#6
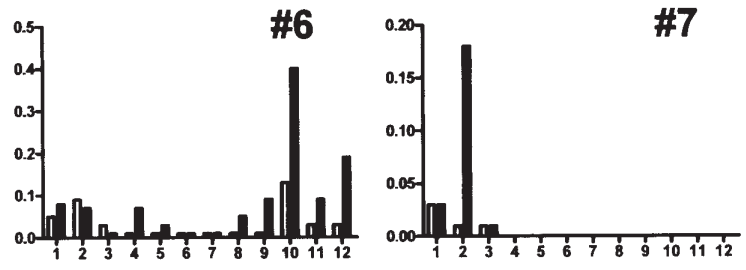

\#8
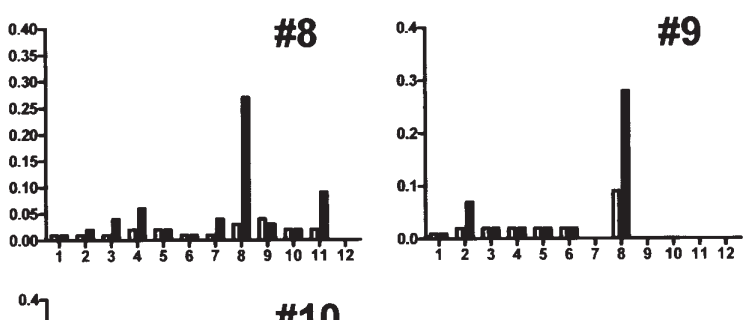

\#
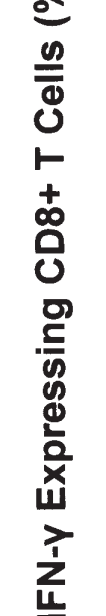

$\$ 9$

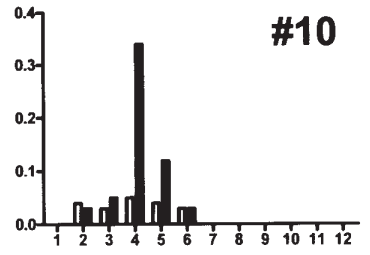

Figure 3. Intracellular IFN- $\gamma$ assay. Blood samples were obtained from patients at different time points and used to detect CD4+ (A) or CD8+ (B) and CD69+ T lymphocytes expressing IFN- $\gamma$ using human IFN- $\gamma$ FastImmune kit (BD Biosciences). The numbers under the $\mathrm{X}$ axis are 1: prior to first vaccine; 2 : two weeks post first vaccine; 3: four weeks post first vaccine; 4: prior to second vaccine; 5: one week post second vaccine; 6: four weeks post second vaccine; 7: prior to third vaccine; 8: one week post third vaccine; 9: four weeks post third vaccine; 10 prior to fourth vaccine; 11: one week post fourth vaccine; 12: four weeks post fourth vaccine. Open bars represent the percentage of IFN- $\gamma$ expressing T cells without activation as controls, while the solid bars represent the percentage of IFN- $\gamma$ expressing $\mathrm{T}$ cells after in vitro activation with tumor lysates.

fusion mixtures were used in those studies. Our animal studies and in vitro human study have shown that highly purified dendritomas formed between DCs and tumor cells maintaining the complete antigenic diversity of the tumor cell population stimulated stronger anti-tumor immune responses $(35,36)$. In this pilot study, 10 stage IV melanoma patients were enrolled to test the safety of this therapy and possible anti-tumor immune responses of the dendritoma vaccine. The results indicated that dendritoma therapy is safe and that there are no significant side effects attributable to the vaccine. All the patients tolerated the vaccine very well. There were some grade 2, 3, and 4 adverse effects observed due to the IL-2 injection and disease progression.

In order to determine if an antitumor immune response is stimulated by the dendritoma vaccine, the number of IFN- $\gamma$ expressing $\mathrm{T}$ cells was measured by FACS analysis for the patients before and after dendritoma vaccine. As shown in Fig. 3, by comparing to the percentage of IFN- $\gamma$ expressing $\mathrm{T}$ cells before vaccine, 8 of 9 evaluable patients showed
IFN- $\gamma$ expressing CD4+ T cell increase at at least one time point, while 6 of 7 analyzed patients showed IFN- $\gamma$ expressing $\mathrm{CD} 8+\mathrm{T}$ cell increase at at least one time point. It is noteworthy that the increase of IFN- $\gamma$ expressing $\mathrm{T}$ cells occurred differently in different patients. Some increases took place as earlier as 2 weeks after the first vaccination, while others happened after second, third, or even fourth vaccination. Although the increase of IFN- $\gamma$ expressing $\mathrm{T}$ cells after dendritoma vaccination did not guarantee a clinical response, all the patients who had complete clinical response (Patient No. 6), stable disease (Patient No. 2 and 9), or mixed response (Patient No. 8) demonstrated increased IFN- $\gamma$ expressing CD4+ T cells and CD8+ T cells at different time points after vaccinations. Although most of the patients (Patient No. 2, 6, 8,9 and 10) showed increases for both IFN- $\gamma$ expressing CD4+ T cells and CD8+ T cells, Patient No. 3 only showed increased IFN- $\gamma$ expressing CD4+ T cells, not CD8+ T cells, while Patient No. 7 showed increased IFN- $\gamma$ expressing CD8+ T cells, not CD4+ T cells. In most of the cases where 
the percentage of IFN- $\gamma$ expressing T cells increased, the percentage of IFN- $\gamma$ expressing T cells in PBMCs that were activated with autologous tumor lysates (solid bars in Fig. 3) is higher than in the IFN- $\gamma$ expressing T cells in PBMCs that were not activated with autologous tumor lysates (open bars in Fig. 3). This demonstrates a tumor cell-specific immune response. These results indicate that the measurement of IFN- $\gamma$ expressing $\mathrm{T}$ cells is a useful monitor of immune responses in cancer immunotherapy and both CD4+ T cells and CD8+ $\mathrm{T}$ cells need to be included. We also observed that the immune responses measured as the increase of IFN- $\gamma$ expressing $\mathrm{T}$ cells after vaccination did not continue in most of the patients, which agrees with the observations from another study (34). Further studies are necessary to understand the mechanism behind this phenomenon.

In most of the melanoma-related vaccine studies which resulted in an anti-tumor immune response, the patients sometimes developed an autoimmune response, such as vitiligo (37-46). To our surprise, we did not observe any autoimmune response after the dendritoma vaccine, even for the patient who had a complete remission. The lack of autoimmune response may be due to the unique characteristics of the dendritomas, which, unlike other vaccines that contain many unfused melanoma tumor cells and DCs or tumor-derived proteins, are purified hybrids of DCs and tumor cells. In this case, since the dendritoma functions as a normal 'professional' antigen presenting cell, normal self-antigens such as melanin, presented in this context, may not elicit a T-cell response, but only the truly tumor unique antigens, many of which have not yet been characterized, may elicit T-cell responses. More studies are needed to confirm this speculation.

IL-2 plays a vital role in activating immune responses because it is required for the growth of T lymphocytes, NK cells, and LAK cells. Preclinical animal studies and human practice have shown that high doses of IL-2 treatments demonstrated some therapeutic anti-tumor effects, but the adverse effect of high dose IL-2 is severe and sometimes is life-threatening. Low dose IL-2 regimens have been tested with limited success. However, when combined with other treatment such as LAK cells, this low dose IL-2 showed some anti-tumor effects (40). Our animal studies also demonstrated that IL-2 treatment alone in the same dose range did not show any anti-tumor effects (data not shown). Yet, when combined with dendritoma treatment, a boosting effect was observed. Therefore, IL-2 was given in a low-dose regimen in this study to boost the antitumor immune responses initiated by dendritomas. Most adverse reactions in this trial were attributable to the IL-2 injections and were recognized side effects of IL-2.

DC-mediated cancer immunotherapy has been tested in clinical trials in many ways including gene transfer, tumor antigen pulsing, and DC-tumor fusions. There is evidence for the induction of tumor-specific anti-tumor immune responses and, in some cases, clinical responses (34,41-46). It remains unclear what is the optimal method for their use. Transfection or transduction of DCs with tumor antigen genes can only deliver very limited tumor antigens. Incubation of DCs with tumor cell lysates can deliver all known and unknown tumor antigens. However, in this way, the tumor antigens will only be processed and presented to CD8+ T cells. DC-tumor fusion represents a better approach for tumor antigen presentation.
The fusion efficiency is relatively low in most of the cases reported, especially for fresh tumor cells. The injection of these DC-tumor fusion mixtures that contain unfused DCs and tumor cells and DC-DC or tumor-tumor self fusion will hinder the activation of functional immune responses. Therefore, as presented in this study, dendritoma therapy, which contains as high as $80 \%$ of purified DC-tumor fused cells, may represent a better approach for cancer immunotherapy.

In conclusion, this study has demonstrated that the dendritoma vaccine can be safely administered to cancer patients and is effective in stimulating immune responses. Although some clinical responses were observed, a larger trial with more patients enrolled is necessary to determine the efficacy of this immunotherapy.

\section{Acknowledgements}

We thank Drs Martin Crane and Dawn Blackhurst of GHS for their help in the data analysis, Kathy Brant and Susan Schultis for their assistance in patient scheduling, and Kim Reiser for her nursing care. We also thank Lakendra Workman for her expert administrative assistance. This study was supported in part by the Oncology Research Foundation of the Greenville Hospital System, Greenville, SC, USA.

\section{References}

1. Steinman R: The dendritic cell system and its role in immunogenicity. Annu Rev Immunol 9: 271-276, 1997.

2. Macatonia S, Taylor P, Knight S, et al: Primary stimulation by dendritic cells induces antiviral proliferative and cytotoxic $\mathrm{T}$ cell responses in vitro. J Exp Med 169: 1255-1264, 1989.

3. Mehta-Damani A, Markowicz S and Engleman E: Generation of antigen-specific CD8+ CTLs from naïve precursors. J Immunol 153: 996-1003, 1994.

4. Porgador A and Gilboa E: Bone marrow-generated dendritic cells pulsed with a class I-restricted peptide are potent inducers of cytotoxic T lymphocytes. J Exp Med 182: 255-260, 1995.

5. Sznol M: Emerging concepts in cancer vaccine development. Principles Pract Oncol 13: 1-14, 1999.

6. Ridgway D: The first 1000 dendritic cell vaccines. Cancer Invest 21: 873-886, 2003.

7. Wei Y, Li J, Chen WY, et al: Enhanced transgene expression and effective in vivo anti-tumor immune response initiated by dendritic progenitors transfected with a nonviral T7 vector expressing a model tumor antigen. J Immunother 23: 75-82, 2000.

8. Stockert E, Jager E, Chen YT, et al: A survey of the humoral immune response of cancer patients to a panel of human tumor antigens. J Exp Med 187: 1349-1354, 1998.

9. Sahin U, Tureci O, Schmitt H, et al: Human neoplasms elicit multiple specific immune responses in the autologous host. Proc Natl Acad Sci USA 92: 11810-11813, 1995

10. Gabrilovich DI, Chen HL, Girgis KR, et al: Production of vascular endothelial growth factor by human tumors inhibits the functional maturation of dendritic cells. Nature Med 2: 1096-1103, 1996.

11. Ishida T, Oyama T, Carbone DP, et al: Defective function of Langerhans cells in tumor-bearing animals is the result of defective maturation from hemopoietic progenitors. J Immunol 161: 4842-4851, 1998.

12. Rosenberg SA, Yang JC, Schwartzentruber DJ, et al: Immunologic and therapeutic evaluation of a synthetic peptide vaccine for the treatment of patients with metastatic melanoma. Nature Med 4: 321-327, 1998.

13. Wallack MK, Sivanandham M, Balch CM, et al: A phase III randomized double-blind multiinstitutional trial of vaccinia melanoma oncolysate-active-specific immunotherapy for patients with stage II melanoma. Cancer 75: 34-42, 1995.

14. Bystryn JC: Clinical activity of a polyvalent melanoma antigen vaccine. Recent Results Can Res 139: 337-348, 1995. 
15. Mitchell MS: Perspective on allogeneic melanoma lysates in active-specific immunotherapy. Semin Oncol 25: 623-635, 1998.

16. Morton DL, Hoon DS, Nizze JA, et al: Polyvalent melanoma vaccine improves survival of patients with metastatic melanoma. Ann NY Acad Sci 690: 120-134, 1993.

17. Berd D, Kairys J and Dunton C: Autologous, hapten-modified vaccine as a treatment for human cancers. Semin Oncol 25: 646-653, 1998.

18. Berd D, Maguire HC Jr, Schuchter LM, et al: Autologous hapten-modified melanoma vaccine as postsurgical adjuvant treatment after resection of nodal metastases. J Clin Oncol 15: 2359-2370, 1997.

19. Abbas AK, Lichtman AH and Pober JS: Cellular and Molecular Immunology. 4th edition. Philadelphia, PA, pp161-180, 2000.

20. Gong J, Chen L, Kashiwaba M, et al: Induction of antigenspecific antitumor immunity with adenovirus-transduced dendritic cells. Gene Ther 4: 1023-1028, 1997.

21. Song W, Kong H, Carpenter H, et al: Dendritic cells genetically modified with an adenovirus vector encoding the cDNA for a model antigen induce protective and therapeutic antitumor immunity. J Exp Med 186: 1247-1256, 1997.

22. Specht J, Wang G, Do M, et al: Dendritic cells retrovirally transduced with a model antigen gene are therapeutically effective against established pulmonary metastases. J Exp Med 186: 1213-1221, 1997.

23. Li J, Holmes LM, Franek KJ, et al: Murine tyrosinase expressed by a T7 vector in bone marrow derived dendritic progenitors effectively prevents and eradicates melanoma tumors in mice. Can Gene Ther 7: 1448-1455, 2000.

24. Trefzer U, Weingart G, Chen Y, et al: Hybrid cell vaccination for cancer immune therapy: first clinical trial with metastatic melanoma. Int J Cancer 85: 618-626, 2000.

25. Gong J, Avigan D, Chen D, et al: Activation of antitumor cytotoxic $\mathrm{T}$ lymphocytes by fusions of human dendritic cells and breast carcinoma cells. Proc Natl Acad Sci USA 97: 2715-2718, 2000.

26. Gong J, Nikrui N, Chen D, et al: Fusions of human ovarian carcinoma cells with autologous or allogeneic dendritic cells induce antitumor immunity. J Immunol 165: 1705-1711, 2000.

27. McConnell EJ, Pathangey LB, Madsen CS, et al: Dendritic celltumor cell fusion and staphylococcal enterotoxin B treatment in a pancreatic tumor model. J Surg Res 107: 196-202, 2002.

28. Xia J, Tanaka Y, Koido S, et al: Prevention of spontaneous breast carcinoma by prophylactic vaccination with dendritic/ tumor fusion cells. J Immunol 170: 1980-1986, 2003.

29. Zhang JK, Li J, Zhang J, et al: Antitumor immunopreventive and immunotherapeutic effect in mice induced by hybrid vaccine of dendritic cells and hepatocarcinoma in vivo. World $\mathrm{J}$ Gastroenterol 9: 479-484, 2003.

30. Marten A, Renoth S, Heinicke T, et al: Allogeneic dendritic cells fused with tumor cells: preclinical results and outcome of clinical phase I/II trial in patients with metastatic renal cell carcinoma. Hum Gene Ther 14: 484-494, 2003.

31. Kawada M, Ikeda H, Takahashi $\mathrm{T}$, et al: Vaccination of fusion cells of rat dendritic and carcinoma cells prevents tumor growth in vivo. Int J Cancer 105: 520-526, 2003.
32. Takana H, Shimizu K, Hayashi T, et al: Therapeutic immune response induced by electrofusion of dendritic and tumor cells. Cell Immunol 220: 1-12, 2002.

33. Chen D, Xia J, Tanaka Y, et al: Immunotherapy of spontaneous mammary carcinoma with fusions of dendritic cells and mucin 1 positive carcinoma cells. Immunology 109: 300-307, 2003.

34. Avigan D, Vasir B, Gong J, et al: Fusion cell vaccination of patients with metastatic breast and renal cancer induces immunological and clinical responses. Clin Cancer Res 10: 4699-4708, 2004.

35. Holmes LM, Li J, Sticca RP, et al: A rapid, novel strategy to induce tumor cell specific cytotoxic T lymphocyte (CTL) responses using instant dendritomas. J Immunother 24: 122-129, 2001.

36. Li J, Holmes LM, Franek KJ, et al: Purified hybrid cells from dendritic cell and tumor cell fusions are superior activators of antitumor immunity. Cancer Immunol Immunother 50: 456-462, 2001.

37. Tsao H, Millman P, Linette G. P, et al: Hypopigmentation associated with an adenovirus-mediated gp 100/MART-1 transduced dendritic cell vaccine for metastatic melanoma. Arch Dermatol 138: 799-802, 2002.

38. Slingluff CL Jr, Petroni GR, Yamshchikov GV, et al: Clinical and immunologic results of a randomized phase II trial of vaccination using four melanoma peptides either administered in granulocyte-macrophage colony-stimulating factor in adjuvant or pulsed on dendritic cells. J Clin Oncol 21: 4016-4026, 2003.

39. Le Poole IC, Wankowicz-Kalinska A, van den Wijngaard RM, et al: Autoimmune aspects of depigmentation in vitiligo. J Investig Dermatol Symp Proc 9: 68-72, 2004.

40. Rosenberg SA: Principles and Practice of the Biologic Therapy of Cancer. 3rd edition. Lippincott Williams and Wilkins, pp50-73, 2000.

41. Nair SK, Morse M, Boczkowski D, et al: Induction of tumorspecific cytotoxic T lymphocytes in cancer patients by autologous tumor RNA-transfected dendritic cells. Ann Surg 235: 540-549, 2002

42. Nestle FO, Alijagic S, Gilliet M, et al: Vaccination of melanoma patients with peptide- or tumor lysate-pulsed dendritic cells. Nat Med 4: 328-332, 1998.

43. Banchereau J, Palucka AK, Dhodapkar M, et al: Immune and clinical responses in patients with metastatic melanoma to CD34 (+) progenitor-derived dendritic cell vaccine. Cancer Res 61: 6451-6458, 2001

44. Timmerman JM, Czerwinski DK, Davis TA, et al: Idiotypepulsed dendritic cell vaccination for B-cell lymphoma: clinical and immune responses in 35 patients. Blood 99: 1517-1526, 2002

45. Schuler-Thurner B, Schultz ES, Berger TG, et al: Rapid induction of tumor-specific type $1 \mathrm{~T}$ helper cells in metstatic melanoma patients by vaccination with mature, cryopreserved, peptide-loaded monocyte-derived dendritic cells. J Exp Med 195: 1279-1288, 2002.

46. Geiger JD, Hutchinson RJ, Hohenkirk LF, et al: Vaccination of pediatric solid tumor patients with tumor lysate-pulsed dendritic cells can expand-specific T cells and mediate tumor regression. Cancer Res 61: 8513-8519, 2001. 